\title{
IBS
}

\section{ROLE OF SODIUM CHANNELS IN IBS}

A potential new pathogenetic mechanism for IBS has been revealed-involving abnormal functioning of the voltage-gated sodium channel $\mathrm{Na}_{\mathrm{v}} 1.5$.

"The roots for this study go back quite a long time," explains Gianrico Farrugia, one of the corresponding authors of this study. Previous investigations had found that a considerable number of patients with cardiac arrhythmias and mutations in SCN5A (which encodes the $\alpha$-subunit of $\mathrm{Na}_{v} 1.5$ ) also had gastrointestinal symptoms. This research was followed up with a pilot study in 50 patients with IBS in which one patient was found to have a mutation in the sodium channel.

In the current study, the researchers used various approaches to investigate their idea that some patients with IBS might carry SCN5A mutations affecting the function of $\mathrm{Na}_{\mathrm{v}}$ 1.5. "We sequenced DNA from patients with IBS and confirmed that mutations resulted in electrophysiological disturbances using patch clamp technology," reports Farrugia. 584 patients with IBS and 1,308 control individuals were included in this part of the study. The results were confirmed in a genome-wide association study and replicated in 1,745 patients in four independent cohorts of patients with IBS and control individuals.

In addition, the researchers focused on one patient who had the most obvious electrophysiological defect and determined the effects of mexiletine (a clinically available drug used to treat cardiac arrhythmias) in vitro and in vivo. Mexiletine was able to restore the sodium current in cells expressing mutant SCN5A from this patient; administration of the drug to the patient (who had constipationpredominant IBS) was found to normalize bowel habits.

The researchers conclude that $\sim 2.2 \%$ of patients with IBS might have an abnormally functioning $\mathrm{Na}_{\mathrm{v}} 1.5$. Farrugia explains that these results build on research in IBS suggesting that components of this disorder have a genetic background. "We are working with investigators at UCLA to replicate our findings," he says. "We are also planning to determine if we can use clinically available drugs to target this ion channel and to treat IBS by taking a disease-modifying approach rather than treating symptoms."

Isobel Leake

Original article Beyder, A. et al. Loss-of-function of the voltage-gated sodium channel $\mathrm{Na}_{\mathrm{v}} 1.5$ (channelopathies) in patients with irritable bowel syndrome. Gastroenterology doi:10.1053/j.gastro.2014.02.054 